\title{
Masas cardíacas, utilidad de la ecocardiografía transesofágica durante el intraperioperatorio
}

\author{
Intracardiac tumors: utility of transesophageal \\ echocardiography during the periodic intraoperative
}

Dominella Fernando', Luis Masco', Longo Silvina²

\begin{abstract}
We present our experience in cardiac tumor resection surgery in adult patients: 30 subjects with sternotomy approach with later diagnosis of myxomas (12), fibroelastomas (7), sarcomas (4), cardiac methastasis of a breast cancer and cardiac invasion of renal tumor (6), and 3 with videothroacoscopic approach with diagnosis of atrial myxoma (2) and intraventricular sarcoma (1). We highlight the usefulness of TEE as an essential monitor in this subtype of cardiac surgery in allowing location confirmation and completion of resection. Likewise as anesthesiologists and active participants of the surgical team, we were able to document absence of residual heart defects, lesions or perforations or dysfunction of heart valves. Evaluation of preexisting anatomy and function and post Cardiopulmonary Bypass ventricular function and circulation were important in early diagnosis of complications.
\end{abstract}

\section{RESUMEN}

Presentamos nuestra experiencia en resección de tumores cardíacos en pacientes adultos: 30 casos con resecciones tumorales por esternotomía: 12 mixomas, 7 fibroeslastomas, 4 sarcomas, 6 tumores renales con invasión cardíaca y 1 metástasis cardíaca de cáncer de mama. En 3 pacientes las resecciones tumorales fueron por videotoracoscopía (2 mixomas y un sarcoma intraventricular). La cirugía por video supone un nuevo desafío para el anestesiólogo.
Key words:

Cardiac tumor, transesophageal echocardiography

Palabras clave:

Tumores cardiacos, ecocardiografía transesofágica

\footnotetext{
Médicos residentes de anestesiología.

Anestesióloga.

Hospital Privado Universitario de Córdoba. Córdoba, Argentina.
}

Fecha de recepción: 24 de noviembre de 2019

Fecha de aceptación: 22 de diciembre de 2019

\section{ORCID}

https://orcid.org/0000-0001-5822-8727

Correspondencia:

Dra. Silvina Longo

Email: silvinalongo@icloud.com 
Destacamos la utilidad de la ecocardiografía transesofágica como monitor, hoy imprescindible en cirugía cardíaca. Éste nos permitió confirmar la localización del tumor y su completa resección. Así mismo, como anestesiólogos y formando parte activa en el equipo quirúrgico, pudimos documentar la ausencia de defectos residuales, perforaciones o incompetencia de las válvulas cardíacas; evaluar la función ventricular y la volemia postcirculación extracorpórea, obtener información no conocida previamente y realizar así un diagnóstico precoz de complicaciones.

\section{Introducción}

as masas cardíacas son formaciones anormales en el interior de las estructuras del corazón o - inmediatamente adyacentes a ellas[1],[2]. Éstas pueden ser clasificadas en tres tipos: trombos, tumores y vegetaciones.

Los trombos intracavitarios constituyen las masas cardiacas más frecuentes. Pueden estar presentes en cualquier cavidad. Entre los factores que predisponen a su aparición se encuentran la estasis sanguínea, anomalías de la motilidad regional (infarto de miocardio, miocardiopatía dilatada), enfermedades inflamatorias, catéteres o dispositivos implantables (Figuras 1a y b). Su ubicación más frecuente es la aurícula izquierda (orejuela) debido fundamentalmente a la alta prevalencia de fibrilación auricular[3] (Figura 2a, b y c). Los trombos en el ventrículo izquierdo se encuen- tran relacionados principalmente al infarto, sobre todo de pared anterior[4],[5]. En cuanto a los trombos de localización derecha se relacionan principalmente a dispositivos implantables, catéteres, dilatación auricular marcada y menos frecuentemente a cardiopatía isquémica[6],[7]. Es importante destacar que en las cavidades derechas pueden encontrarse trombos "en tránsito" correspondientes a tromboembolismo pulmonar (Figuras 3a y b). Estos también pueden ser observados en la arteria pulmonar y sus ramas[8].

Los tumores cardíacos primitivos son raros: su frecuencia es de aproximadamente 0,02\%[9]-[10]. Se originan principalmente del tejido conectivo. El $75 \%$ son benignos y el $25 \%$ malignos[9],[10]. La sintomatología que producen depende primariamente de la localización (pericárdica, intramural, endocavitaria) de la cámara afectada, del tamaño y de su naturaleza infiltrante.
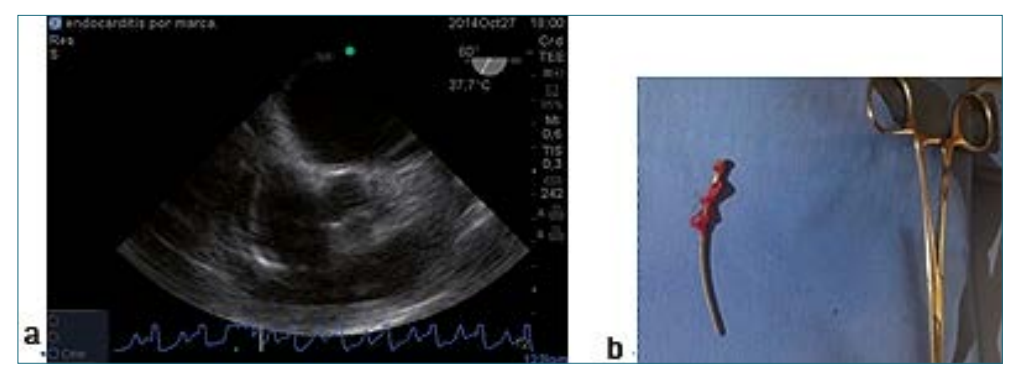

Figura 1. a) Imagen a $60^{\circ}$ medioesofágica: donde se observa cable de marcapasos en aurícula derecha con endocarditis; b) pieza quirúrgica endocarditis sobre el cable retirado.
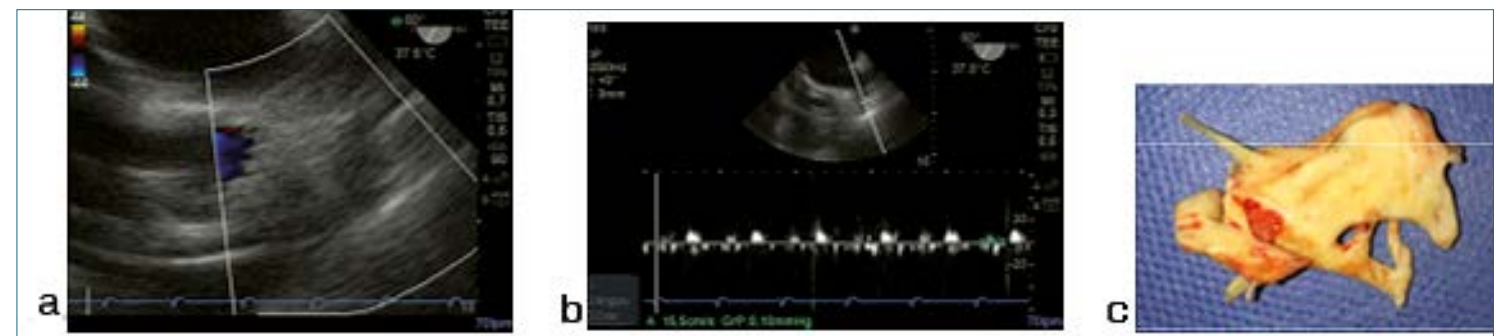

Figura 2. a) Zoom en orejuela de aurícula izquierda, doppler color negativo; b) Doppler pulsado velocidad baja $15 \mathrm{~cm} / \mathrm{s}$; c) Trombo resecado de orejuela de aurícula izquierda. 


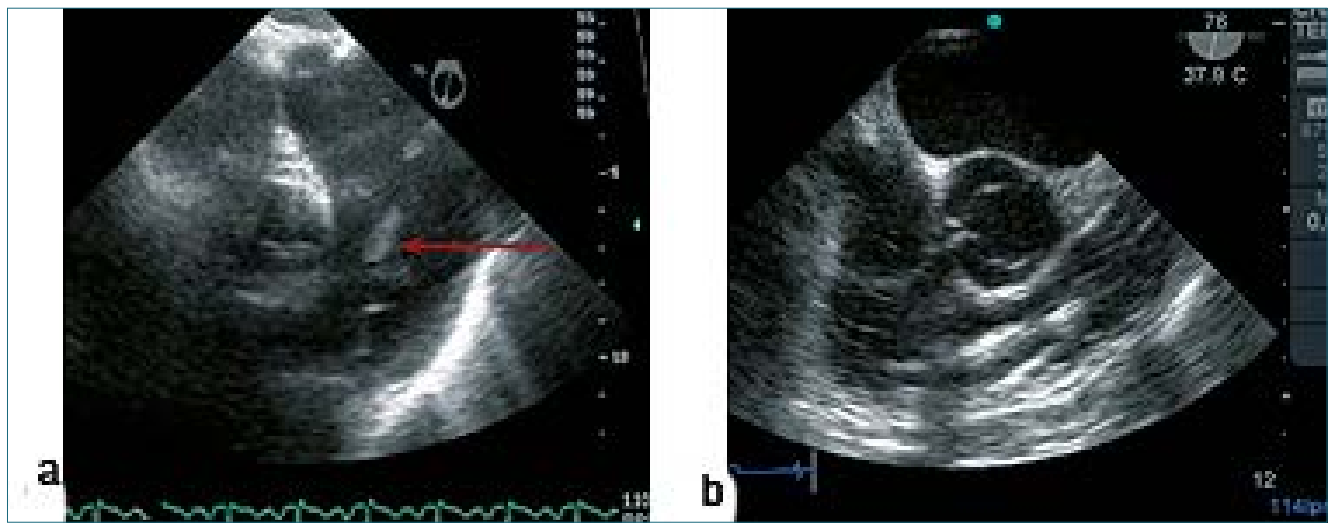

Figura 3. a) Imagen a $76^{\circ}$ esofágica alta donde se ve trombo en arteria pulmonar (flecha); b) imagen a $76^{\circ}$ tracto de entrada y salida de ventrículo derecho (VD), trombo en curso en VD hacia arteria pulmonar.
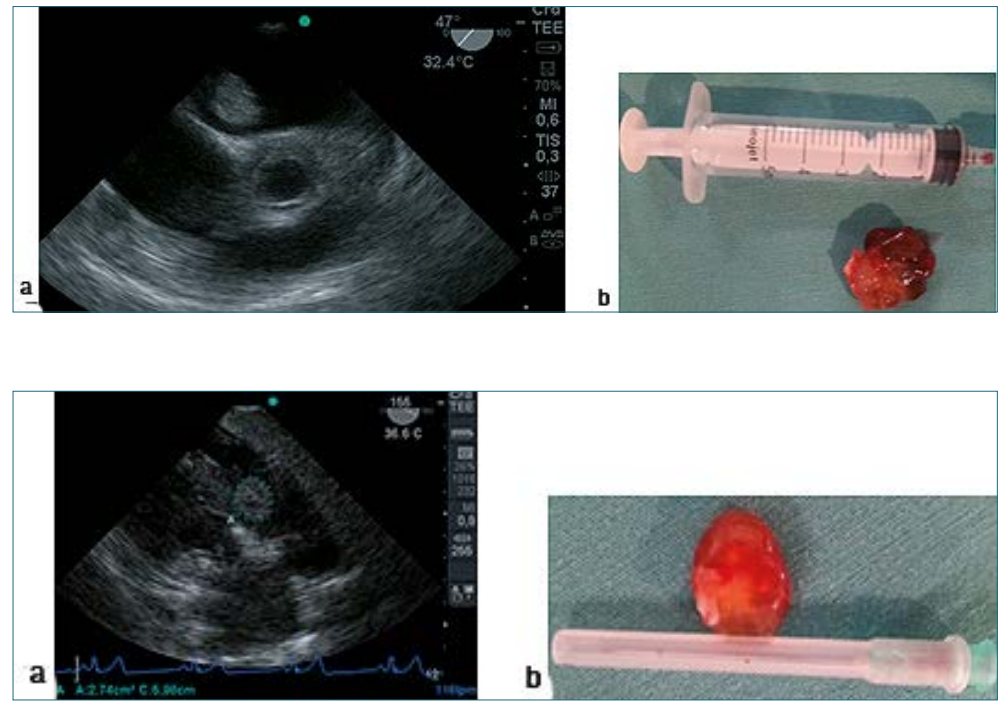

Figura 4. a) Imagen a $47^{\circ}$ medioesofágica, tumor en aurícula izquierda (Al); b) Mixoma de Al.

Figura 5. a) Imagen transgástrica a $155^{\circ}$, tumor en aurícula derecha (AD) sobre valva septal de la tricúspide; b) pieza quirúrgica de mixoma $A D$
Los tumores intracavitarios son más sintomáticos que los intramurales, ya que pueden producir interferencia con el funcionamiento valvular o generar fenómenos embólicos[11]. Por su localización, pueden producir obstrucción del flujo, embolismos o arritmias severas.

El mixoma es el tipo de tumor cardíaco primario más frecuente. Representa el $50 \%$ de los tumores benignos y el $25 \%$ del total[12],[13],[14]. Es más frecuente en el sexo femenino y la edad media de diagnóstico es alrededor de los 50 años. Existe una forma esporádica (la más frecuente) y otra familiar. Más del $90 \%$ son solitarios. La localización más frecuente es la aurícula izquierda (83\%)[12],[13],[14],[15], anclados en la pared medial de la cavidad (Figuras $4 a$ y b).
Luego siguen en frecuencia los mixomas auriculares derechos (Figuras 5 a y b). La ubicación biauricular representa $1 \%$ de los casos. Otras ubicaciones como los ventrículos, las válvulas o la vena cava inferior son inusuales. Macroscópicamente se presentan como masas blandas, pediculadas, de consistencia friable y colorido grisáceo. Pueden tener áreas de trombosis o hemorragia en su interior. Crecen rápidamente y miden habitualmente de 4 a $8 \mathrm{~cm}$. Los síntomas generados por los mixomas pueden ser sistémicos (por la producción de mediadores químicos o la embolización) o locales (disnea de esfuerzo, sincope, muerte súbita).

El fibroelastoma papilar es un tumor benigno poco frecuente. Su prevalencia real se desconoce, pero se 


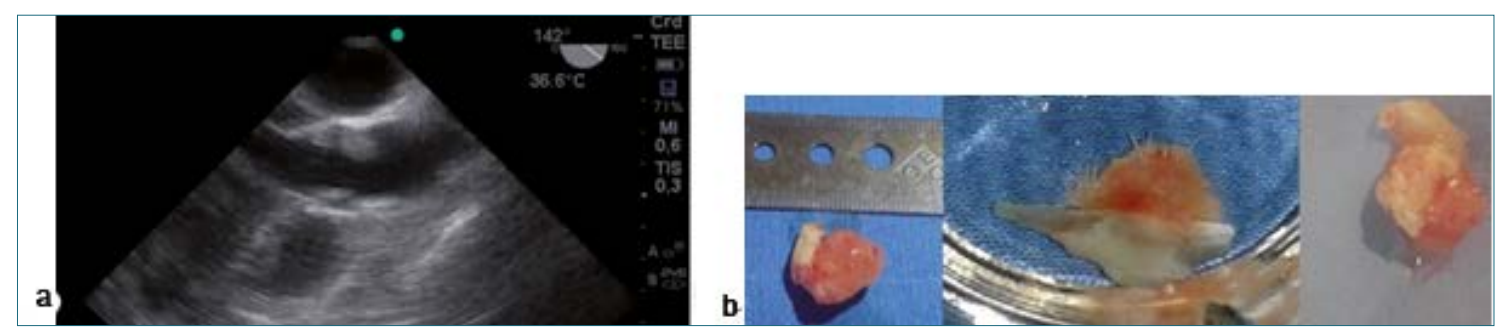

Figura 6. a) Imagen a $142^{\circ}$ medioesofágico: eje largo de válvula aórtica (VA), tumor en valva coronariana izquierda; b) fibroelastoma de VA colocado en agua para ver su aspecto velloso.

estima en alrededor de 0,2\%[16]. Su localización principal es el endocardio valvular, principalmente izquierdo, con predilección de la válvula aórtica. Constituyen el tumor valvular más frecuente[17],[18]. Estos tumores tienen una apariencia gelatinosa, friable, grisácea. Suelen ser pequeños (alrededor de $1 \mathrm{~cm}$ ). Cuando se sumergen en un medio líquido, adquieren aspecto de medusa[19]. Estas pequeñas excresencias suelen ser asiento de trombos, los cuales embolizan distalmente, siendo ésta la principal manifestación clínica de los fibroelastomas (Figura 6).

Existen otros tumores benignos, menos frecuentes, como el mesotelioma del nodo auriculoventricular: se expresa clínicamente con bloqueo auriculoventricular y es el tumor benigno que produce muerte súbita con mayor frecuencia. Otro tumor benigno poco frecuente es el quiste pericárdico, que es el tumor pericárdico más frecuente. Habitualmente afectan el lado derecho del corazón y pueden llegar a medir $15 \mathrm{~cm}$.

Los lipomas cardíacos pueden ser intracavitarios, intramiocárdicos o intrapericárdicos. Los subepicárdicos suelen ser grandes y pueden provocar ángor por compresión de las arterias coronarias.

Los hemangiomas pueden asentarse en el epicardio o el miocardio, ya sea en el ventrículo izquierdo o derecho. Las manifestaciones clínicas incluyen arritmias, derrame pericárdico, insuficiencia cardíaca e incluso muerte súbita[20]. Se presentan como intramurales o intracavitarios.

La utilización del ecocardiograma transesofágico (ETE), por su proximidad con el corazón y la ventaja de no tener interpuesto el pulmón y los huesos, nos brinda imágenes de gran calidad, y representa una herramienta fundamental en el intraoperatorio. Nos permite realizar una valoración integral del paciente, evaluar la función sistólica y diastólica, determinar la localización del tumor, afectación de las válvulas cardíacas, y luego de la resección documentar la ausencia de defectos residuales como comunicaciones interauriculares o perforaciones valvulares.

\section{Métodos}

Analizamos los pacientes sometidos a cirugía cardíaca para resección tumoral en nuestra institución en el período de 2013 a 2019, un total de 30 pacientes, a los cuales, previo consentimiento informado, se les realizó ETE intraoperatorio. Ningún paciente padecía patología esofágica o gástrica severa que contraindicara su colocación.

Todos los pacientes contaban con el diagnóstico preoperatorio de tumor cardíaco realizado con resonancia magnética y ecocardiografía transtoráxica (ETT) en la valoración preoperatoria.

Previo a la inducción de la anestesia general se realizó un acceso venoso periférico y arterial para medición de presión arterial invasiva. Después de la inducción anestésica se colocó una vía central yugular interna derecha y la sonda de ETE sin dificultades.

\section{Resultados}

Un total de 30 pacientes portadores de tumores cardíacos fueron monitorizados con ETE entre los años 2013 a 2019 (Tabla 1).

En todos los casos se insertó el transductor sin dificultad y se lograron obtener imágenes de excelente calidad.

El principal motivo para monitorizar a los pacientes fue la visualización del tumor, su tamaño, localización y compromiso de otras estructuras cardíacas. Además, se buscó comprobar post CEC la resección en su totalidad, la evaluación de la precarga, la reposición con fluidos, la función ventricular sistólica y diastólica, comprobar los resultados terapéuticos, detectar isquemia antes que otros monitores, descubrir complicaciones y patologías desconocidas previamente.

En once pacientes el tumor fue mixoma en aurícula izquierda, cuya localización más frecuente fue la 


\section{Tabla 1. Frecuencia de tumores cardíacos en la serie de casos}

\section{Tumores primarios benignos}

Mixoma 12 pacientes: 11 en aurícula izquierda y 1 en aurícula derecha

Fibroelastoma 7

\section{Tumores malignos}

Tumor renal con invasión cardíaca 6

Sarcoma 2

Mesotelioma pericárdico 2

Metástasis de cáncer de mama 1

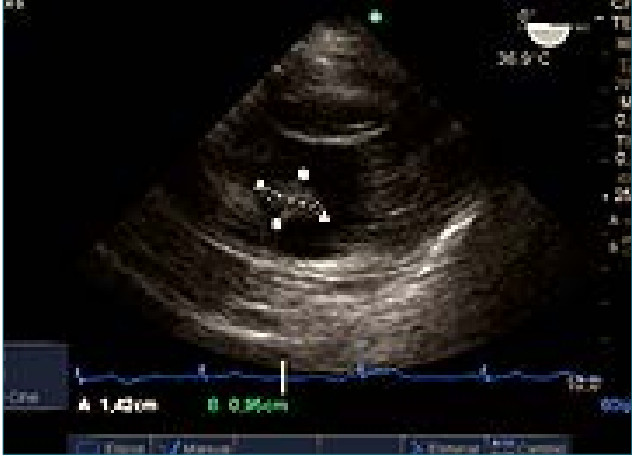

Figura 7. Imagen a $0^{\circ}$ transgástrica basal eje corto, tumor en el borde libre (A2).

pared auricular y el septo (Figura 1). En todos los casos con ETE se corroboró la resección total del tumor y la ausencia de comunicación auricular realizando la prueba del pasaje de burbujas inyectadas a presión por el catéter de presión venosa central (PVC) con una mezcla de sangre del propio paciente y solución fisiológica (20 ml) (Figura 11). Se consideró que el tabique se encontró indemne si en los próximos 3 latidos no se detectó presencia de burbujas en aurícula izquierda.

Un paciente se diagnosticó como mixoma auricular derecho adherido a la valva septal de la válvula tricuspídea (Figura 5). Posterior a la salida de circulación extracorpórea (CEC) se comprobó la ausencia de insuficiencia tricuspídea para lo cual se utilizó doppler color, medición de vena contracta (menor a $3 \mathrm{~mm}$ ) y la morfología normal con doppler pulsado en venas suprahepáticas (ausencia de inversión de la onda sistólica "s"). El paciente era portador de cardiopatía isquémica, con ETE se realizó diagnóstico de isquemia del territorio de la arteria coronaria derecha y circunfleja a la salida de CEC que requirió apoyo de inotrópicos y vasodilatadores coronarios hasta la completa recuperación de la función miocárdica.

Siete pacientes fueron portadores de fibroelastoma. Cuatro de ellos, de la válvula aórtica (Figura 6). Se comprobó el correcto funcionamiento valvular, la ausencia de insuficiencia (observada con doppler pulsado y color a $120^{\circ}$ desde la imagen medioesofágica de 3 cámaras) tracto de salida aórtico; y se descartó estenosis aórtica, medida realizada desde la imagen transgástrica profunda con medición del área valvular y de los gradientes máximo y medio transvalvular aórtico. Una vez extraída la pieza se realizó la confirmación del tipo de tumor al colocarlo en agua y observar la característica vellosa de un tumor en apariencia inicial liso. Los otros tres pacientes tenían el diagnóstico de tumor adherido a la válvula mitral con sospecha de mixoma versus fibroelastoma. Con ETE se localizó el tumor en el borde libre de A2 de la valva anterior de la mitral (Figura 7). El tumor se resecó sin lesión valvular
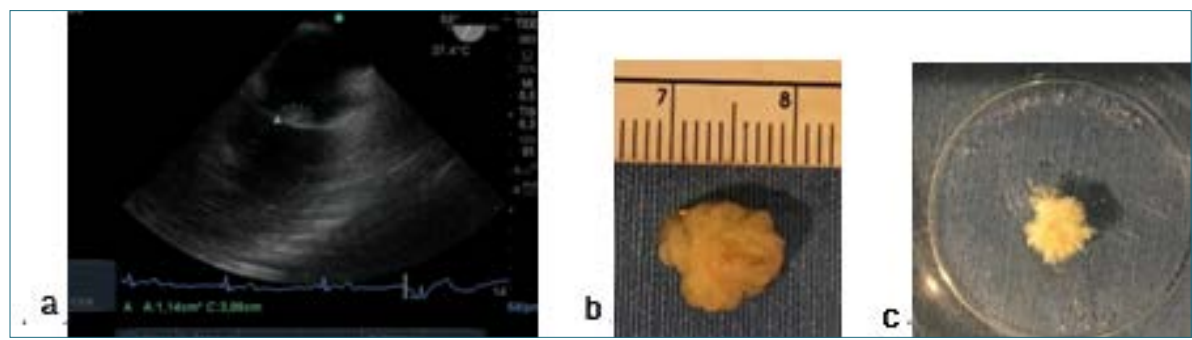

Figura 8. a) Imagen a $60^{\circ}, 2$ cámaras medioesofágica: a-tumor en cara auricular de válvula mitral; b) Fibroelastoma; c) tumor colocado en agua. 

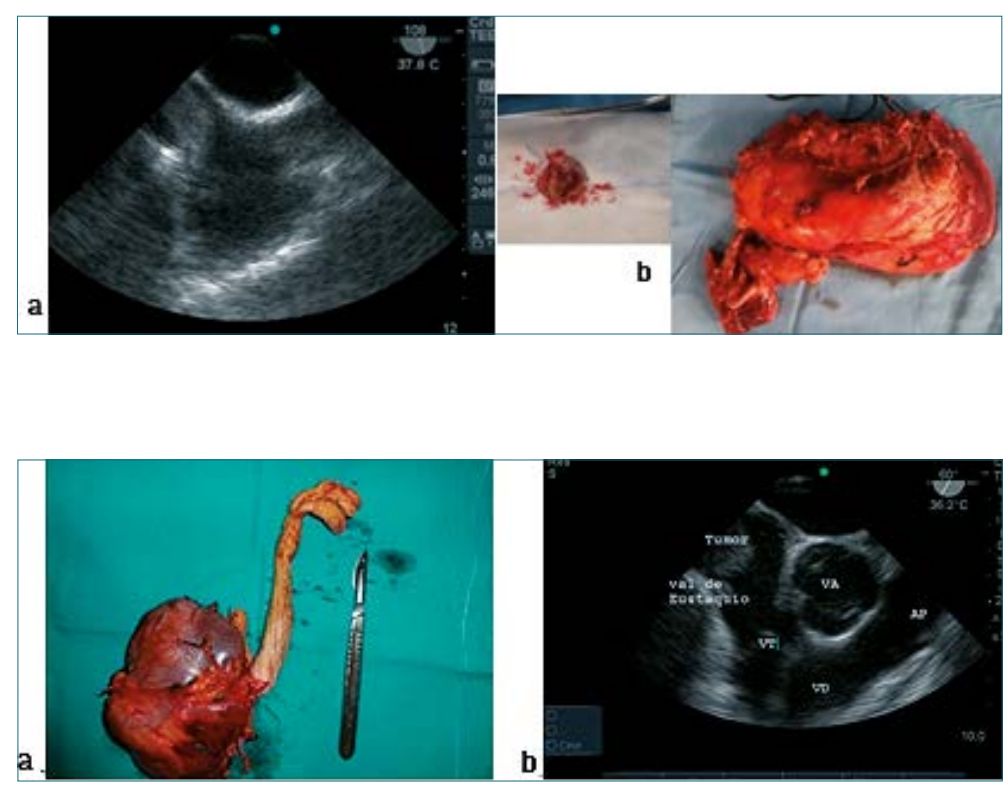

Figura 10. a) Pieza quirúrgica riñón y tumor renal; b) Imagen medioesofágica a $60^{\circ}$, eje corto de válvula aórtica: tumor renal en vena cava inferior hasta aurícula derecha.

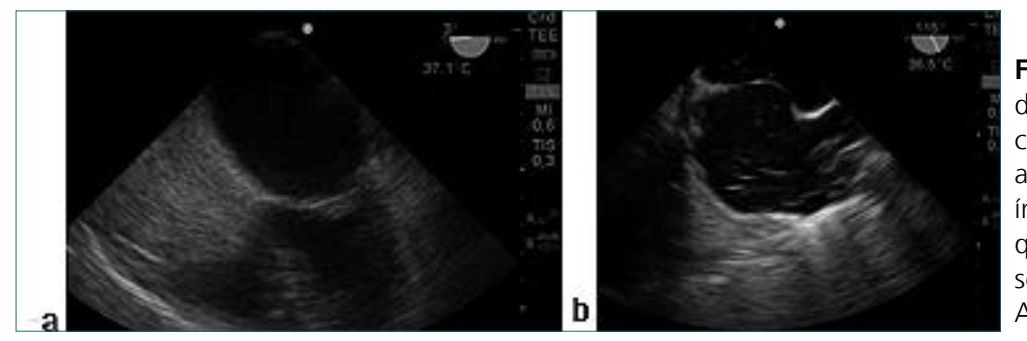

Figura 11. a) Imagen medioesofágica de 4 cámaras $0^{\circ}$ opacificación de aurícula derecha sin pasajede burbujas hacia aurícula izquierda. Tabique interauricular íntegro; b) Imagen bicava a $115^{\circ}$, tabique interauricular aneurismático, se observa pasaje de burbujas desde AD hacia Al.

y luego el aspecto velloso del tumor en agua realizó el diagnóstico de fibroelastoma (Figuras $8 \mathrm{~b}$ y c). La recuperación del corazón y la ausencia de defectos residuales fueron guiadas por ecocardiografía.

Siete pacientes ingresaron para resección de tumor renal con invasión de vena cava inferior que ascendía hasta la aurícula derecha (Figuras 9 y 10). Un tumor metástasis auricular derecha de cáncer de mama. ETE fue guiando la ubicación precisa del nivel alcanzado, asegurando la resección total y guiando la recuperación cardíaca post CEC, luego de una cirugía con parada circulatoria.

Cuatro pacientes presentaron tumores malignos de ventrículo izquierdo, 2 intracavitarios y 2 localizados en la cara pericárdica del músculo. Estos últimos se presentaron clínicamente con derrame pericárdico hemorrágico. El ETE permitió evaluar la magnitud de la invasión al músculo cardíaco sano y guiar la resección quirúrgica.

\section{Discusión}

Los tumores cardíacos primarios son extremadamente raros, presentan una incidencia de menos del $0,1 \%[9],[10]$. En contraparte, la afectación metastásica del corazón es 20 veces más frecuente y se ha reportado en series de autopsias, en hasta uno de cada cinco pacientes que mueren de cáncer[21],[22]. Los tumores cardíacos primarios se clasifican en benignos y malignos. Más de $75 \%$ de ellos son benignos. En adultos la gran mayoría son mixomas; le siguen en frecuencia, el fibroelastoma papilar, fibromas y lipomas.

Entre el $15 \%$ y el $25 \%$ restante son tumores malignos, siendo los sarcomas los más frecuentes. En nuestra serie 4 pacientes presentaron tumores malignos primarios. De los 30 pacientes 8 fueron metástasis.

El ETE como monitor hemodinámico ha significado un gran avance en medicina, permitiendo visuali- 

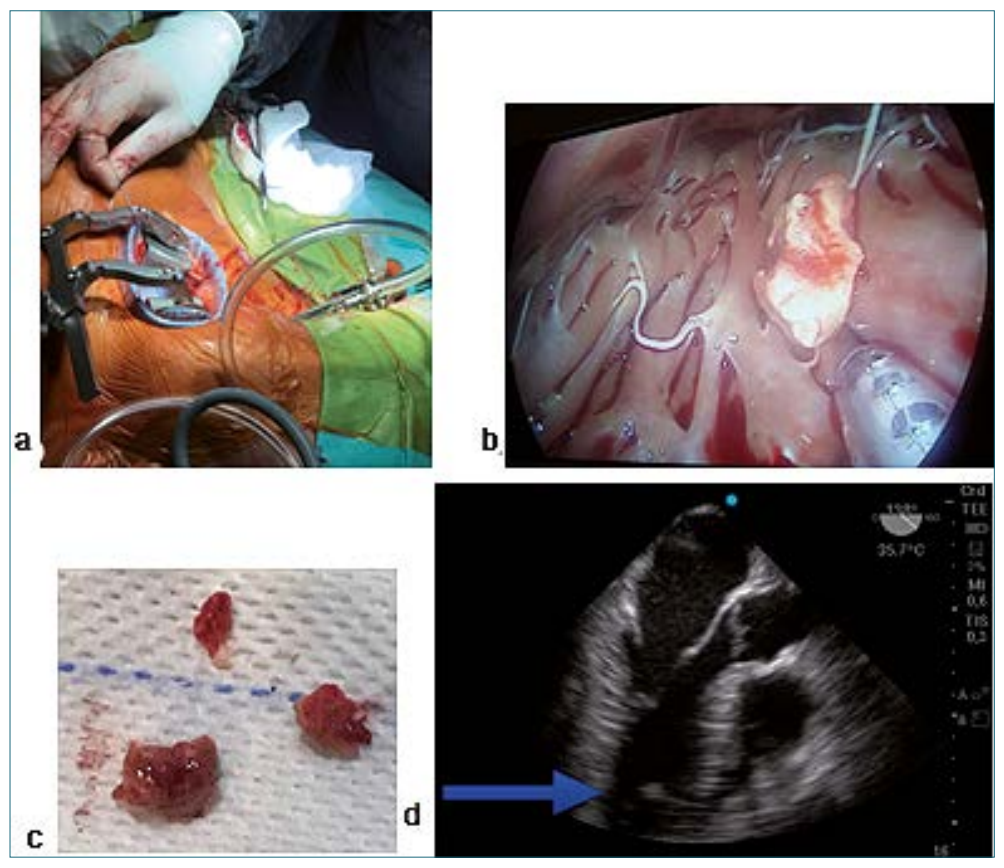

Figura12. a) Incisión torácica y videocámara; b) imagen de video con tumor ventricular; c) Pieza quirúrgica; d) imagen de 3 cámaras $M E$, la flecha señala tumor de VI.
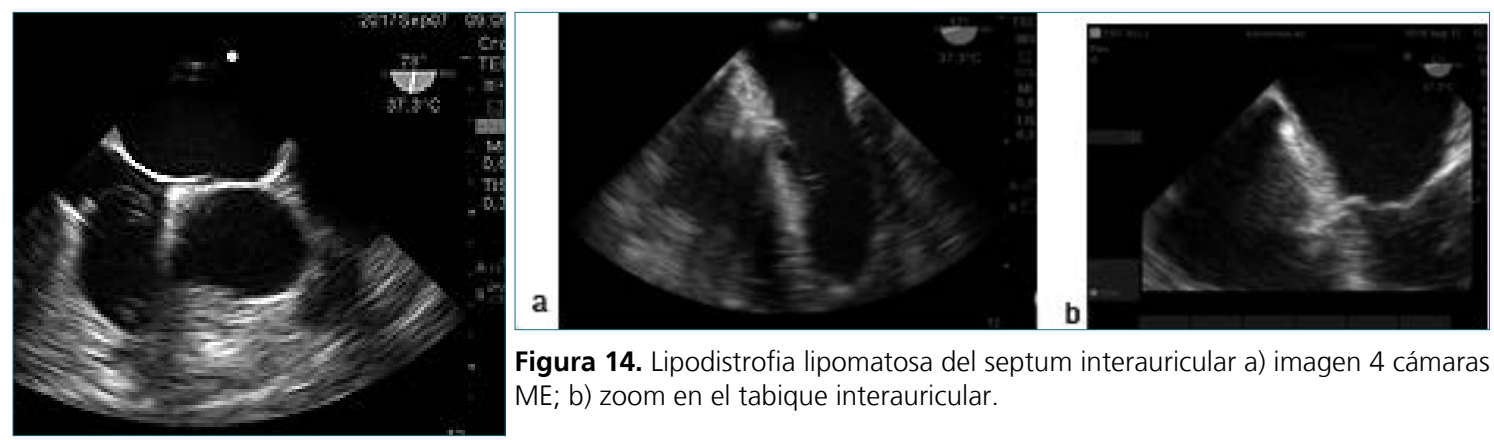

Figura 14. Lipodistrofia lipomatosa del septum interauricular a) imagen 4 cámaras $\mathrm{ME} ;$ b) zoom en el tabique interauricular.

Figura 13. Válvula de Eustaquio en la desembocadura de la VCI en la AD.

zar en forma directa y en tiempo real el corazón y los grandes vasos, observando el impacto del tumor en el contexto general cardíaco. Poder evaluar en forma directa la precarga, la contractilidad y la postcarga del miocardio, son valiosos datos que nos permiten conocer exactamente el estado actual y la fisiopatología cardíaca.

Durante la realización de ETE algunas estructuras cardíacas "normales" no deben confundirnos con la presencia de tumores. Muchas de estas estructuras son remanentes embrionarios, variantes normales, que deben ser diferenciados para la correcta valoración de los pacientes (Tabla 2, Figuras 13, 14 y 15).
Sin bien estos tumores pueden causar múltiples signos y síntomas, consecuencia generalmente de la localización[23], en la mayoría de los casos el diagnóstico fue incidental, esto en gran parte debido al mayor control clínico de los pacientes y el acceso a estudios complementarios como el ETT. Pueden producir obstrucción del flujo, al interferir con las válvulas cardíacas, la invasión de estructuras como el miocardio, pericardio y pulmón con la pleura, lo que produciría arritmias y derrames. También embolización o producción de síntomas constitucionales[24],[25].

Es por esta variada sintomatología cardíaca que se los llama "grandes imitadores". Los mixomas; simu- 


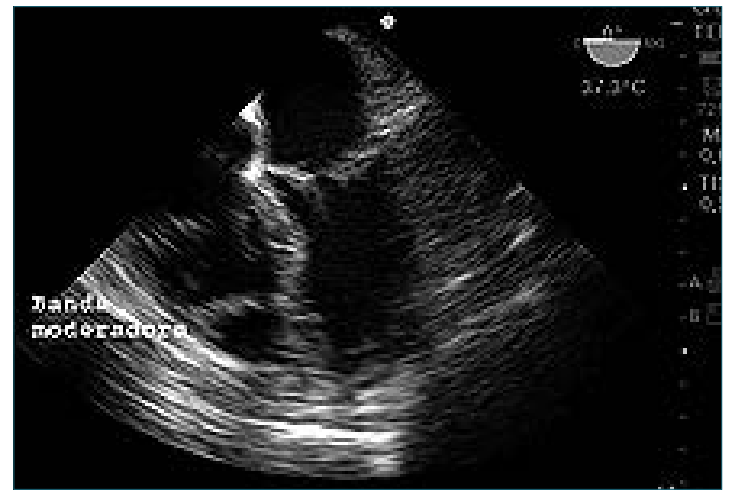

Figura 15. Imagen de 4 cámaras ME banda moderadora en VD.

lan enfermedades valvulares, en ocasiones el tumor se comporta como una estenosis mitral o tricúspide, cuando al nacer de las paredes auriculares crecen obstruyendo la circulación sanguínea durante la diástole, los fibroelastomas simulan vegetaciones, muchas veces el diagnóstico de tumor se realiza en el estudio de un paciente con diagnóstico de embolia posiblemente cardíaca, y los fibromas, rabdomiomas y sarcomas pueden confundirse con miocardiopatías hipertróficas localizadas[26].

Se realizaron 3 casos de resección de tumor por videotoracoscopía. Esta técnica se asocia con menores complicaciones en comparación con la técnica habitual (esternotomía). La cirugía cardíaca por videotoracoscopía impone nuevos desafíos para el anestesiólogo y nos impone un rol activo; realizamos bloqueo de los erectores de la espina (ESP) para lograr adecuada analgesia postoperatoria. Se requiere intubación selectiva con tubo de doble lumen, la canulación venosa se realiza mediante control ecocardiográfico transesofágico.
Este abordaje resulta en extubación temprana, menor cantidad de días de internación en cuidados intensivos, reducción de las infecciones, disminución de complicaciones respiratorias y se evita dolor prolongado postoperatorio.

\section{Conclusión}

El diagnóstico de tumores es cada vez más temprano y certero debido a la consulta temprana y los métodos de diagnóstico disponibles. Razón por la cual muchos tumores se operan en estadios temprano y en pacientes asintomáticos. La evolución de la cirugía cardíaca y la anestesia general junto a la información que aportó ETE es de gran utilidad no solo en la resección del tumor, sino que nos fue guiando en la completa recuperación cardíaca, obtuvimos valores de volúmenes, gradientes y presiones de las cámaras cardíacas, medición del volumen sistólico y del gasto cardíaco. Aseguramos la ausencia de defectos residuales cardíacos como resultado de la resección de tumores adheridos a paredes auriculares, válvulas cardíacas o pared de grandes vasos como $\mathrm{VCl}$.

Destacamos los beneficios de la cirugía por videotoracoscopia que como cirugía cardiovascular mínimamente invasiva coloca al anestesiólogo en un rol activo, debiendo realizar múltiples procedimientos invasivos[28]. Esta técnica permite lograr un mayor confort postoperatorio para el paciente y permite disminuir las complicaciones postoperatorias.

El diagnóstico de los tumores intracardíacos ha evolucionado enormemente en los últimos años, debido al desarrollo del diagnóstico por imágenes. En el presente trabajo comunicamos nuestra experiencia y valoramos que ETE representa una herramienta fundamental en la valoración intraoperatoria de los pacientes sometidos a cirugía para resección tumoral.

\begin{tabular}{l} 
Tabla 2 \\
\hline Estructuras normales que se pueden confundir con tumor \\
1- Válvula de Eustaquio grande \\
2- Crista terminalis \\
3- Red de Chiari \\
4- Banda moderadora del ventrículo derecho \\
5- Músculos papilares \\
6- Músculos pectíneos en la orejuela de ambas aurículas \\
7- Lipodistrofia del tabique interauricular \\
\hline
\end{tabular}




\section{Referencias}

1. Ragland MM, Tak T. The role of echocardiography in diagnosing space-occupying lesions of the heart. Clin Med Res. 2006 Mar;4(1):22-32. https:// doi.org/10.3121/cmr.4.1.22 PMID:16595790

2. Auger D, Pressacco J, Marcotte F, Tremblay A, Dore A, Ducharme A. Cardiac masses: an integrative approach using echocardiography and other imaging modalities. Heart. 2011 Jul;97(13):1101-9. https://doi. org/10.1136/hrt.2010.196006 PMID:21642661

3. DePace NL, Soulen RL, Kotler MN, Mintz GS. Two dimensional echocardiographic detection of intraatrial masses. Am J Cardiol. 1981 Nov;48(5):954-60. https://doi.org/10.1016/00029149(81)90364-7 PMID:7197875

4. Stratton JR, Lighty GW Jr, Pearlman AS, Ritchie JL. Detection of left ventricular thrombus by twodimensional echocardiography: sensitivity, specificity, and causes of uncertainty. Circulation. 1982 Jul;66(1):156-66. https://doi. org/10.1161/01.CIR.66.1.156 PMID:7083502

5. Keren A, Goldberg S, Gottlieb S, Klein J, Schuger C, Medina A, et al. Natural history of left ventricular thrombi: their appearance and resolution in the posthospitalization period of acute myocardial infarction. J Am Coll Cardiol. 1990 Mar;15(4):790-800. https://doi.org/10.1016/07351097(90)90275-T PMID:2307788

6. Chartier L, Béra J, Delomez M, Asseman P, Beregi JP, Bauchart $\mathrm{J}$, et al. Free-floating thrombi in the right heart: diagnosis, management, and prognostic indexes in 38 consecutive patients. Circulation. 1999 Jun;99(21):277983. https://doi.org/10.1161/01.
CIR.99.21.2779 PMID:10351972

7. Stowers SA, Leiboff RH, Wasserman AG, Katz RJ, Bren GB, $\mathrm{Hsu}$ I. Right ventricular thrombus formation in association with acute myocardial infarction: diagnosis by 2-dimensional echocardiography. Am J Cardiol. 1983 Oct;52(7):912-3. https://doi.org/10.1016/00029149(83)90442-3 PMID:6624686

8. Kessler KM, Mallon SM, Bolooki $\mathrm{H}$, Myerburg RJ. Pedunculated right ventricular thrombus due to repeated blunt chest trauma. Am Heart J. 1981 Dec;102(6 Pt 1):1064-6. https://doi.org/10.1016/00028703(81)90492-0 PMID:7315703

9. Comess KA, DeRook FA, Russell $M L$, Tognazzi-Evans TA, Beach $\mathrm{KW}$. The incidence of pulmonary embolism in unexplained sudden cardiac arrest with pulseless electrical activity. Am J Med. 2000 Oct;109(5):3516. https://doi.org/10.1016/ s0002-9343(00)00511-8 PMID:11020390

10. McAllister HA, Fenoglio JJ. Tumors of the cardiovascular system. Atlas of Tumor Pathology (2nd series, fasc. 15). Washington (DC): Armed Forces Institute of Pathology; 1978. pp. 1-3.

11. García Gallego F, Gamallo Amat C, Aguado MG. Registro Nacional de Tumores Cardíacos (memoria 1996-1997). Rev Esp Cardiol. 1999 Jan;52(1):1320. https://doi.org/10.1016/ S0300-8932(99)74859-1 PMID:9989132

12. Burke A, Jeudy J Jr, Virmani R. Cardiac tumours: an update: Cardiac tumours. Heart. 2008 Jan;94(1):117-23. https://doi. org/10.1136/hrt.2005.078576 PMID:18083956

13. Reynen K. Cardiac myxomas. N Engl J Med. 1995
Dec:333(24):1610-7.

https://doi.org/10.1056/

NEJM199512143332407

PMID:7477198

14. Casey M, Mah C, Merliss AD, Kirschner LS, Taymans SE, Denio $A E$, et al. Identification of a novel genetic locus for familial cardiac myxomas and Carney complex. Circulation. 1998 Dec;98(23):2560-6. https://doi. org/10.1161/01.CIR.98.23.2560 PMID:9843463

15. Fisher J. Cardiac myxoma. Cardiovasc Rev Rep. 1983;9:1195-9.

16. Dujardin KS, Click RL, Oh JK. The role of intraoperative transesophageal echocardiography in patients undergoing cardiac mass removal. J Am Soc Echocardiogr. 2000 Dec;13(12):1080-3. https://doi. org/10.1067/mje.2000.107157 PMID:11119275

17. Klarich $\mathrm{KW}$, Enriquez-Sarano M, Gura GM, Edwards WD, Tajik AJ, Seward JB. Papillary fibroelastoma: echocardiographic characteristics for diagnosis and pathologic correlation. J Am Coll Cardiol. 1997 Sep;30(3):784-90. https://doi.org/10.1016/S07351097(97)00211-8 PMID:9283541

18. Sun JP, Asher $C R$, Yang $X S$, Cheng GG, Scalia GM, Massed AG, et al. Clinical and echocardiographic characteristics of papillary fibroelastomas: a retrospective and prospective study in 162 patients. Circulation. 2001 Jun;103(22):2687-93. https://doi.org/10.1161/01.

CIR.103.22.2687 PMID:11390338

19. Cook AG, Viswanath $O$, D'Mello J. Papillary Fibroelastoma Found With Transesophageal Echocardiography After a Normal Transthoracic Echocardiography. Semin Cardiothorac Vasc Anesth. 2017 Sep;21(3):217-20. https://doi. rg/10.1177/1089253217699281 
PMID:28758563

20. Patel J, Patel S, Sheppard MN. Benign cardiac tumours associated with sudden death. Europace. 2014 Jun;16(6):855-60. https://doi.org/10.1093/europace/eut378 PMID:24390387

21. Reynen K. Frequency of primary tumors of the heart. Am J Cardiol. 1996 Jan;77(1):107. https://doi.org/10.1016/ S0002-9149(97)89149-7 PMID:8540447

22. Salcedo EE, Cohen GI, White RD, Davison MB. Cardiac tumors: diagnosis and management. Curr Probl Cardiol. 1992 Feb;17(2):73-137. https:// doi.org/10.1016/01462806(92)90025-J PMID:1735241

23. Vander Salm TJ. Unusual primary tumors of the heart. Semin Thorac Cardiovasc Surg. 2000 Apr;12(2):89-100. https://doi. org/10.1053/ct.2000.5080 PMID:10807431

24. Elbardissi AW, Dearani JA, Daly RC, Mullany CJ, Orszulak TA, Puga FJ, et al. Embolic potential of cardiac tumors and outcome after resection: a case-control study. Stroke. 2009 Jan;40(1):156-62. https:// doi.org/10.1161/STROKEAHA.108.525709 PMID:18948602

25. Sheu CC, Lin SF, Chiu CC, Lee CS, Chai CY, Liu YC, et al. Left atrial sarcoma mimicking obstructive pulmonary disease. J Clin Oncol. 2007 Apr;25(10):1277-9. https://doi. org/10.1200/JCO.2006.09.9077
PMID:17401018

26. Smith M, Chaudhry MA, Lozano $\mathrm{P}$, Humphrey MB. Cardiac myxoma induced paraneoplastic syndromes: a review of the literature. Eur J Intern Med. 2012 Dec;23(8):669-73. https://doi. org/10.1016/j.ejim.2012.05.015 PMID:23122392

27. Shanmugam G. Primary cardiac sarcoma. Eur J Cardiothorac Surg. 2006 Jun;29(6):925-32. https://doi. org/10.1016/j.ejcts.2006.03.034 PMID:16675225

28. Parnell $A$. and Prince $M$. Anaesthesia for minimally invasive cardiac surgery. BJA Education, 18(10): 323e330 (2018) https://doi.org/10.1016/j. bjae.2018.06.004 